

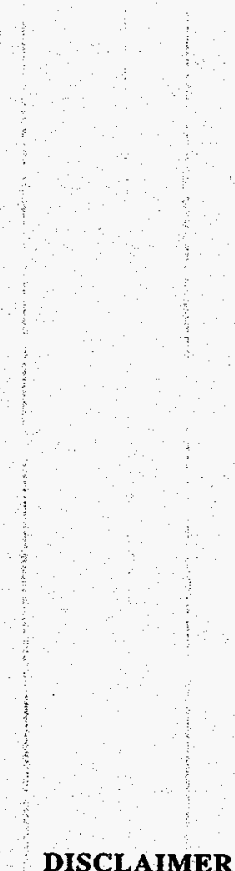

This document was prepared as an account of work sponsored by the United States Government. While this document is believed to contain correct information, neither the United States Government nor any agency thereof, nor The Regents of the University of California, nor any of their employees, makes any warranty, express or implied, or assumes any legal responsibility for the accuracy, completeness, or usefulness of any information, apparatus, product, or process disclosed, or represents that its use would not infringe privately owned rights. Reference herein to any specific commercial product, process, or service by its trade name, trademark, manufacturer, or otherwise, does not necessarily constitute or imply its endorsement, recommendation, or favoring by the United States Government or any agency thereof, or The Regents of the University of California. The views and opinions of authors expressed herein do not necessarily state or reflect those of the United States Government or any agency thereof, or The Regents of the University of California.

Available to DOE and DOE Contractors

from the Office of Scientific and Technical Information

P.O. Box 62, Oak Ridge, TN 37831

Prices available from (615) $576-8401$

Available to the public from the

National Technical Information Service

U.S. Department of Commerce

5285 Port Royal Road, Springfield, VA 22161

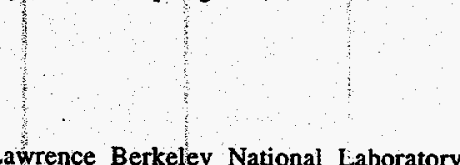

Ernest Orlando Lawrence Berkeley National Laboratory

is an equal opportunity employer. 
LBL-38399

UC-419

HIFAN 803

Life on the Edge: Squirrel-Cage Fringe Fields and Their Effects in the MBE-4 Combiner Experiment

\author{
William M. Fawley \\ Accelerator \& Fusion Research Division \\ Lawrence Berkeley National Laboratory \\ University of California \\ Berkeley, California 94720
}

*Work supported by the Director, Office of Energy Research, Office of Fusion Energy, U.S. Dept. of Energy under Contract No. DE-AC03-76SF00098 at LBL. 


\title{
Life on the Edge: Squirrel-Cage Fringe Fields and Their Effects in the MBE-4 Combiner Experiment*
}

\author{
William M. Fawley \\ Lawrence Berkeley Laboratory, University of California \\ Berkeley, CA 94720 USA
}

\section{Abstract}

The MBE-4 combiner experiment employs an electrostatic combined-function focusing/bending element, the so-called "squirrel-cage" just before the actual merging region. There has been concern that non-linear fields, primarily in the fringe regions at the beginning and end of the cage, may be strong enough to lead to significant emittance degradation. This note present the results of numerical calculations which determined the anharmonic, non-linear components of the $3 \mathrm{D}$ fields in the cage and the resultant, orbit-integrated effects upon the MBE-4 beamlets. We find that while the anharmonic effects are small compared to the dipole deflection, the resultant transverse emittance growth is significant when compared to the expected value of the initial emittance of the individual beamlets.

\section{Introduction}

The present MBE-4 combiner experiment was designed to examine many of the physics and engineering issues associated with the transverse combining and merging of multiple, space-charge-dominated, heavy-ion beams. In order to be a "good" model of a driver combiner, it is important that the relative size of various effects in the MBE-4 experiment be appropriately scaled from what is expected in a driver. One such effect is the emittance growth in the bending and focusing optics of the combiner. This must be compared with both the upstream beam emittance and the growth expected from the merging process downstream of the combiner. Of particular concern in the MBE-4 experiment are the anharmonic fields present in the "squirrel-cage" combined-function focusing/bending element, the last in the combiner section. This element has four transport channels (the "cages"), one for each of the MBE-4 beamlets (see Fig. 1). Each cage comprises approximately twenty, small diameter, conducting wires (some prefer using the word "rods" but due to their small diameter I prefer "wires"). The upstream end of each wire is held by an insulating, ceramic plate and connected via a lead to a voltage divider network, while the downstream end is freely suspended in space. Presently (early 1996), details of the exact path the leads follow from the voltage divider to the upstream end of each cage remain undetermined, as will their effect, if any, on the beam particle dynamics. However, the cages themselves are composed of straight wire elements.

- Work supported by the Director, Office of Energy Research, Office of Fusion Energy, U.S. Dept. of Energy under Contract No. DE-AC03-76SF00098 at LBL
The remainder of this note consists of the following sections: $\$ 2$ describes the cage geometry and the methods for determining the necessary voltages on the individual wires. $\$ 3$ introduces the capacitance matrix and multipole decomposition methods in order to determine the strengths of the fringe and interior anharmonic fields while $\S 4$ presents the actual numerical decomposition results for the top and right cages versus $z$. In $\$ 5$ we calculate the cumulative anharmonic effects by integrating them in $z$ along the nominal trajectory of the beam centroid. The concluding section, $\$ 6$, compares these results with some previous analytical and simulation work on the MBE-4 squirrel-cage.

\section{Wire, Charge, and Electrostatic Field Distribution in the MBE-4 Squirrel-Cage}

\subsection{Adopted Geometry}

As mentioned in the Introduction, the full geometry of the squirrel-cage element in the MBE-4 combiner experiment remains somewhat tentative. For the analysis presented in this note, we adopted the configuration depicted in Fig. 1 and include only the straight section of each wire. The transverse (i.e. $x-y$ ) cross-section of the ensemble (here the transverse origin corresponds to the symmetry center of the four cage ensemble) is tapered and shrinks selfsimilarly with $z$ from the upstream end to the downstream end, i.e.

$$
\left.[x, y]\right|_{z}=\left[x_{d}, y_{d}\right] \times\left(1+\zeta\left(\frac{z-z_{d}}{z_{u}-z_{d}}\right)\right) \quad z_{\imath} \leq z \leq z_{d}
$$

where the subscripts $u$ and $d$ refer to the upstream and downstream quantities respectively. The scaling factor $\zeta$ is determined by the requirement that the outermost wire (whose downstream position is $20.5 \mathrm{~mm}$ from the origin) expand from the origin with a slope equivalent to a 6degree angle $(\approx 0.1$ radian $)$. Hence,

$$
\zeta=\frac{L_{W} \times \tan 6^{\circ}}{y_{\max }}=0.403
$$

for $L_{w}=78.6 \mathrm{~mm}$, the cages's length in $z$. W. Ghiorso kindly provided a file containing the downstream transverse coordinates of each wire and a simple conversion program determined the $(x, y, z)$ coordinates of the discrete charge locations along each wire for purposes of capacitance matrix inversion and multipole decomposition (see $\S$ III). 
The voltages on each wire were calculated following the reasoning of A. Falten's[1]. Within each of the four cages, the vacuum electrostatic field must include both a dipole component to bring $\left\langle x^{\prime}\right\rangle$ and $\left\langle y^{\prime}\right\rangle$ to zero at the end of the squirrel-cage where the four beams actually begin to merge and a quadrupole component to keep the beam envelopes in approximate equilibria with their upstream values.

The squirrel-cage quadrupole field gradient was determined by setting the phase advance of the beam to that necessary to bring the calculated envelope parameters $\left\langle a^{\prime}\right\rangle$ and $\left\langle b^{\prime}\right\rangle$ of the individual beams to zero at the exit of the squirrel-cage. According to the latest envelope code calculations provided by $P$. Seidl, the average quadrupole field gradient must be $18.1 \mathrm{~V} / \mathrm{mm}^{2}$, presuming an effective length of $78.6 \mathrm{~mm}$.

To lowest order (i.e. ignoring the taper of the wires, the approximately parabolic orbit of each beam's centroids, the effects of both edge fringe fields at the cage ends and those due to the discrete nature of the wires azimuthally), the required dipole field is

$E_{D}=\frac{\Delta x^{\prime}}{\Delta z} \times 2 V_{B}=\frac{\tan 6^{\circ}}{78.6 \mathrm{~mm}} \times 400 \mathrm{kV}=5.35 \times 10^{5} \mathrm{~V} / \mathrm{m}$

This field may be produced by displacing the centroid quadrupole field toward the transverse origin of the complete ensemble and away from the geometric centroid of each wire cage. Using the aforementioned quadrupole gradient and expression (3), the required quadrupole field centroid displacement must then be $29.5 \mathrm{~mm}$ from the beam centroid position in each cage. With the nominal, downstream centroid position of the right (vertically-elongated) beam lying at $\left(x_{c}, 0.0\right)$ and that of the top (horizontallyelongated) beam at $\left(0.0, y_{c}\right)$, the wanted potential functions in the right and top cages are

$$
\begin{gathered}
\Phi_{\text {right }}(x, y)=9.0\left[-\left(x+29.5-x_{c}\right)^{2}+y^{2}\right]+\Phi_{r}^{\circ} \\
\Phi_{\text {top }}(x, y)=9.0\left[x^{2}-\left(y+29.5-y_{c}\right)^{2}\right]+\Phi_{t}^{\circ}
\end{gathered}
$$

with all positions measured in $\mathrm{mm}$. The constants $\Phi_{r}^{\circ}$ and $\Phi_{t}^{o}$ are found (or more exactly, their relative difference) by realizing $\Phi_{\text {right }}(x, y)$ must be identical to $\Phi_{\text {top }}(x, y)$ on the three electrodes they share along the line

$$
y=x+3.0 \mathrm{~mm}
$$

Setting $[\hat{x}+\hat{y}] \cdot \vec{\nabla} \Phi_{\text {right }}=[\hat{x}+\hat{y}] \cdot \vec{\nabla} \Phi_{\text {top }}$ along this line forces

$$
y_{c}-x_{c}=6.0 \mathrm{~mm}
$$

With $y_{c}=12.5 \mathrm{~mm}$, then $x_{c}=6.5 \mathrm{~mm}$ which differs slightly from Falten's choice of $x_{c}=6.0 \mathrm{~mm}$. If relations (6) and (7) are inconsistent, this results in much larger anharmonic forces, especially those associated with a sextupole component. Substituting (7) into relations (4) and (5), we find $\Phi_{r}^{o}-\Phi_{t}^{o}=1.08 \mathrm{kV}$. Finally, forcing the voltage at position $(0.0,3.0) \mathrm{mm}$ to be exactly zero then determines the "absolute" values of $\Phi_{r}^{\circ}$ and $\Phi_{t}^{\circ}$ as 4.66 and 3.58 $\mathrm{kV}$ respectively.

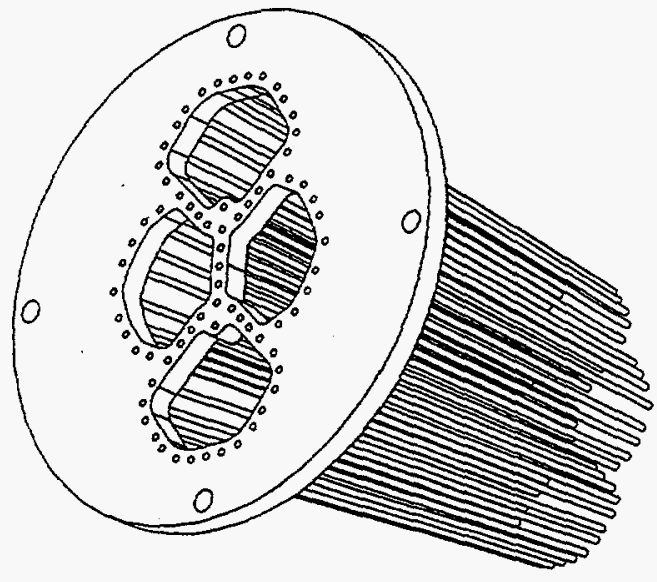

Figure 1: Illustration of the squirrel-cage geometry, showing the upstream insulating ceramic plate and the 71 individual wires and their taper toward the cage center from the upstream to the downstream end.

\section{2 "Higher Order" Analytical Solution for Wire Potentials}

Primarily because the taper with $z$ in the transverse locations of the wires affects the effective length of the quadrupole component with a different power of $(1+\zeta)$ than it does the dipole component, it is prudent to calculate a somewhat more exact solution to the wire potentials. Moreover, the approximately parabolic orbit of the beam centroid also tends to lower the effective dipole deflection than would be calculated from expression (3) because on the average each beam is closer to the ensemble origin than would be true for an exactly linear path with $z$.

Once again ignoring fringe fields, the quadrupole field gradient is given approximately by

$$
E_{Q}^{\prime}(z) \approx E_{q}^{\prime}\left(z_{d}\right) \times\left(1+\alpha\left(z_{d}-z\right)\right)^{-2}
$$

where the fields at $z=z_{d}$ are evaluated without fringe field effects and $\alpha \equiv \zeta /\left(z_{d}-z_{u}\right)$. Integrating in $z$, the average quadrupole gradient through the cage is then

$$
<E_{Q}^{\prime}>\approx E_{q}^{\prime}\left(z_{d}\right) \times \frac{1}{(1+\zeta)}
$$

Hence one must increase the quadrupole component of the wire potentials by a factor of $\approx 1.4$ to $25.3 \mathrm{~V} / \mathrm{mm}^{2}$ to take into account the reduced effectiveness caused by the taper.

The reduction in the effective dipole field is somewhat more complicated because both the variation in $E_{Q}^{\prime}$ and the variation with $z$ in the beam centroid offset $y_{c}$ from the quadrupole centroid $y_{Q}$ must be considered. Analytically,

$$
\begin{array}{r}
E_{D}(z) \approx E_{D}\left(z_{d}\right) \times\left(1+\alpha\left(z_{d}-z\right)\right)^{-1}+ \\
\left(y_{c}(z)-y_{Q}(z)\right) E_{Q}^{\prime}(z)
\end{array}
$$

Here $y_{c}(z)=y_{c}\left(z_{d}\right)+1 / 2 \times y_{u}^{\prime}\left(z_{d}-z\right)^{2}$ and $y_{Q}(z)=$ $y_{Q}\left(z_{d}\right) \times\left[1+\alpha\left(z_{d}-z\right)\right]$. The first term in expression (10) 
reduces the dipole effective length by about $16 \%$ whereas (after a fair amount of boring algebra) the second term is equivalent to an average offset of the beam centroid of $0.8 \mathrm{~mm}$ and thus reduces the dipole effective length by an additional $\approx 3 \%$. Consequently, the net effect of the taper is to reduce the estimated required quadrupole offset (at $z=z_{d}$ ) from 29.5 to $25.7 \mathrm{~mm}$.

\subsection{Numerical Determination of Wire Po- tentials}

The analytical solution presented above still neglects effects from the up- and downstream fringe fields, which due to both the tapered geometry and the offset angle of the cage centroid, will modify the effective lengths of both the dipole and quadrupole fields. For purposes of guiding the MBE-4 experimentalists, we used the multipole method to be discussed in the next solution to obtain a far more accurate solution. By iterating on both $V_{q}$ and the quadrupole offset $y_{Q}\left(z_{d}\right)$, the dipole and quadrupole fields can be adjusted until the wanted integrals are satisfied:

$$
\begin{gathered}
\int E_{D}\left(z^{\prime}\right) d z^{\prime}=\Delta x^{\prime} \times 2 V_{B}=41.8 \mathrm{kV} \\
\int E_{Q}^{\prime}\left(z^{\prime}\right) d z^{\prime}=18.1 \mathrm{~V} / \mathrm{mm}^{2} \times 78.6 \mathrm{~mm}=1.43 \mathrm{kV} / \mathrm{mm}
\end{gathered}
$$

where the field values are calculated along the parabolic path of the beam centroid. Using the "higher order" solution of the last section as our starting point, we found that setting $E_{Q}^{\prime}\left(z_{d}\right)=27.9 \mathrm{~V} / \mathrm{mm}^{2}$ and $y_{Q}\left(z_{d}\right)=21.6 \mathrm{~mm}$ reproduced relations (12) to within $1.5 \%$ and (11) to within $0.7 \%$ in both the top and right cages. These values of $E_{Q^{\prime}}$ and $y_{Q}$ are about $11 \%$ higher and $16 \%$ lower respectively than the "improved" (i.e. taper-cognizant) analytic solution of the last section.

The "ultimate" solution would be to replace $z^{\prime}$ in the last two expressions by $s$ - the actual path - and evaluate both $E_{Q}^{\prime}$ and $E_{D}$ in the non-paraxial limit. Given the total neglect of the effects of the upstream wire leads, we did not feel that the additional programming effort was sufficiently worthwhile. Hence, all the results of the following sections presumed that the orbit of each beam centroid was linear outside the cage extent in $z$ and exactly parabolic inside, with no non-paraxial effects either in the orbit or in the multipole expansion. We expect that the greatest deviation from these approximations will be upstream where $\left\langle x^{\prime}\right\rangle$ is largest.

\section{Electrostatic Potential Solution and Multipole Decomposition}

Since the individual wire voltages are provided by timeinvariant power supplies and we are neglecting fields due to the ion beam, we may take $\mathbf{E} \equiv-\vec{\nabla} \Phi$. If we model the continuous surface and line charge densities by a finite number of discrete point charges, the electrostatic potential at any given point $\mathbf{r}$ may be mathematically expressed by

$$
\Phi(\mathbf{r})=\sum_{j} \frac{Q_{j}}{\left|\mathbf{r}-\mathbf{r}_{j}\right|}
$$

where for convenience we have taken $4 \pi \epsilon_{0} \equiv 1$. In order to determine the values of the $Q_{j}$, one must find the capacity matrix $C_{i j}$ where

$$
Q_{i}=\sum_{j=1}^{j=N} C_{i j} \Phi_{j}
$$

with the elements of the inverse matrix $C^{-1}$ given by

$$
\left[C^{-1}\right]_{i j}=1 / \max \left(\left|\mathbf{r}_{i}-\mathbf{r}_{j}\right|, r_{0}\right)
$$

$Q_{i} / r_{0}$ represents the effective self-potential of the charge $Q_{i}$.

One's choice of the exact value of $r_{0}$ depends upon the nature of the problem (e.g. surface charges versus line charges) and spatial resolution (higher resolution generally implies a smaller value for $r_{0}$ ). For the current problem of the squirrel-cage, we used a constant spacing $\Delta s$ between adjacent charges on a given wire and chose the value

$$
r_{0} \equiv\left(\frac{1}{4 \ln 2}\right) \Delta s=0.3607 \Delta s=0.45 \mathrm{~mm}
$$

for $\Delta s=1.25 \mathrm{~mm}$. For an isolated, infinitely long wire parallel to the $z$ axis with equal charges at positions $z=$ $\pm k \Delta s, k=0,1,2, \ldots$, this choice sets $\Phi(z=n \Delta s)=\Phi(z=$ $(m+1 / 2) \Delta s)$. Although the exact value of $r_{0}$ has a small effect on the spatial structure of the potential far from individual wires, it will affect the structure within a couple $\Delta s$ of any individual wire.

A small set of numerical experiments involving varying the value of $r_{0}$ showed that for a series of charges equally spaced in azimuth and length (i.e. $r \Delta \theta=\Delta s$ ) the best choice for $r_{0}$ is $0.25 \Delta s$. "Best" was defined as that which, for a test case of an azimuthally uniformly-spaced, 32-wire single cage with comparably-sized monopole through dodecapole potential components, gave a numerical charge solution whose resulting potential multipole decomposition closely (better than $1 \%$ ) reproduced the chosen wire values. When $\Delta s$ exceeds $r \Delta \theta$ by a relatively small factor $\leq 2$, it appears $r_{0}$ should be close to (but somewhat larger than) the 0.25 times the geometric mean of $\Delta s$ and $r \Delta \theta$. For the particular case of the tapered squirrel-cage, the average value of $r \Delta \theta$ is about $2.2 \mathrm{~mm}$ while 0.25 times the geometric mean is $0.41 \mathrm{~mm}$ which is pleasantly close to our value of $r_{o}=0.45 \mathrm{~mm}$.

We wrote a simple FORTRAN code to set up the positions of the individual charges and the resultant inverse capacity matrix. Inasmuch as the inverse matrix is symmetric (i.e. $\left[C^{-1}\right]_{i j}=\left[C^{-1}\right]_{j i}$ ), this permits a somewhat more rapid solution than would be true for the more general case. We chose the IMSL routines LCHRG, PERMU, 
and LFSDS to perform the inversion. A $2520 \times 2520$ matrix (2-mm discrete charge spacing) required 1.5 minutes CPU time (single-processor equivalent) on the NERSC Cray-2 $\mathrm{C}$ machine whereas a $4544 \times 4544$ matrix (1.25-mm spacing) took about 49 seconds on the C90. With normal memory size limitations (in interactive mode) on the NERSC Cray2 's preventing direct inversion of matrices greater than about $3000 \times 3000$ in size and $6000 \times 6000$ in the $C 90$, a different method or algorithm must be used if much greater resolution is sought for this problem. Once the capacitance matrix is found, one directly obtains the corresponding individual charge values for any arbitrary set of wire voltages. Then, from expression (13), the potential at any point may be determined.

The anharmonic components of the electrostatic fields can be found via multipole decomposition. We used a method based on Hahn's[2] algorithm, itself an improvement over that of Berz et al. [3]. When doing this decomposition, we used a "moving" origin in the $x-y$ plane which followed the nominal parabolic path (i.e. that corresponding to a constant $E_{D}$ ) of the beam centroid within each cage. However, the multipole decomposition was always done in the $x-y$ plane exactly perpendicular to the "true" $z$-axis rather than perpendicular to the beam centroid's instantaneous velocity vector. In the absence of voltage or alignment errors, symmetry arguments imply that the the multipole decomposition of the left cage is identical to that of the right cage (and that of the top to that of the bottom) apart from a sign change in the dipole. Following the notation of [2], the harmonic portion of the potential in the $x-y$ plane at a given $z$ may then be expressed

$$
\begin{array}{r}
\Phi_{H A R}(x, y, z) \equiv \quad M_{0,0}(z)+M_{1,1}(z) x+N_{1,1}(z) y+ \\
M_{2,2}(z)\left[x^{2}-y^{2}\right]+N_{2,2}(z) 2 x y(17)
\end{array}
$$

where $x$ and $y$ are measured relative to the moving origin. The non-linear, anharmonic portion of the potential is thus

$$
\Phi_{N L}(x, y, z)=\Phi(x, y, z)-\Phi_{H A R}(x, y, z)
$$

This portion of the potential can produce significant emittance growth depending upon the size of the beam and whether there is significant cancellation of the upstream fringe fields by those downstream.

\section{Numerical Results for the Line Charge and Multipole Distribu- tions versus $\mathrm{z}$}

Once the capacity matrix is determined, one uses expression (14) to find the discrete charges at each of the node locations. Figure 2 plots the charge distribution versus $z$ for selected wires belonging to the top cage for a tapered geometry cases using the numerical solution of the previous section. For this and the following figures, we used a 1.25 -mm spacing in $z$ for the discrete charge representation of the continuous line charge distribution along the

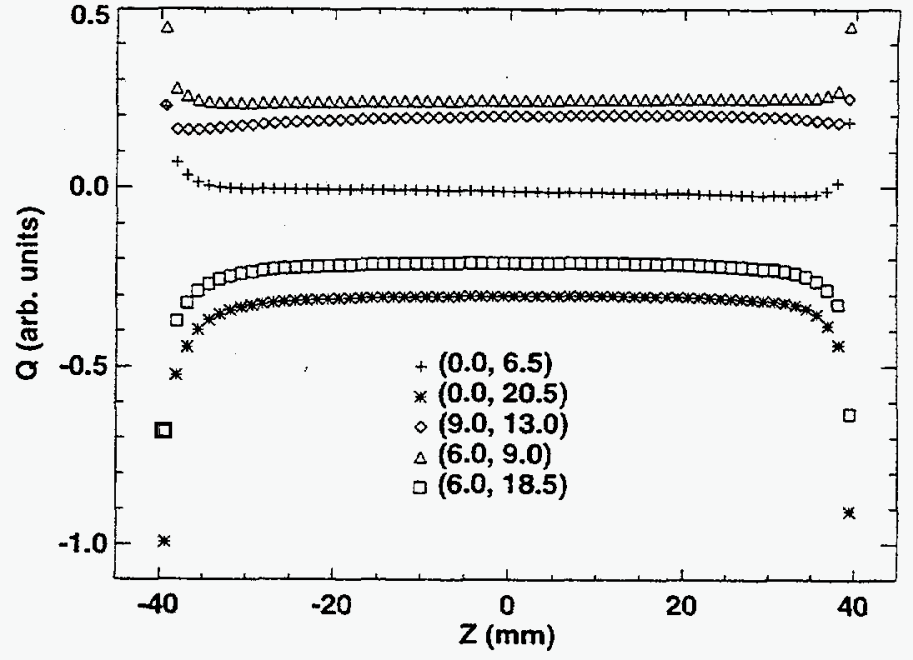

Figure 2: Discrete line charge values along 5 wires in the top cage in a tapered geometry. The numbers within the parentheses refer to the final, downstream $(x, y)$ positions in $\mathrm{mm}$. Apart from the approximately $2 \mathrm{X}$ enhancements at the wire ends, the equivalent line charge density is nearly constant along each wire.

individual wires. One sees that over the bulk of the length of each wire, the line charge density is nearly constant implying that a local 2-D approximation gives a reasonably good answer. Beginning at about $10 \mathrm{~mm}$ from each end, the line charge density begins to change strongly leading to a point charge on the end of each wire whose value is about 2-3 times greater than that of charges deep in the interior. An equivalent plot (not shown) for the untapered geometry is quite similar, with the line charge being nearly constant over the length of a wire.

There is a much greater difference in the top cage multipole distributions versus $z$ between the tapered and untapered cases. For the untapered case (Fig. 3), the decomposition was done along a path that was parabolic within the cage beginning at with the $x-y-z$ coordinate $(0$, $14.84,-35.0) \mathrm{mm}$ and ending at $(0,11.16,35.0) \mathrm{mm}$. Outside the cage, the path was a straight-line with $y^{\prime}$ equal to -0.105 upstream and exactly zero downstream of the cage. The $y$ starting point for the parabola was chosen to approximately balance the sextupole components at the upstream and downstream ends of the cage (as opposed to an alternative choice of ending the parabola at $y=12.5 \mathrm{~mm}$, the approximate vertical midpoint of the top cage. One sees that in addition to the dominant (and wanted) dipole (N11) and quadrupole (M22) terms, there are also sextupole (N33), octupole (M44), and dodecapole (M66) terms that are smaller by a factor of about 100 in terms of their contribution to the local electrostatic potential at $r=5 \mathrm{~mm}$. This smallness confirms that the number of wires used per cage and their placement is sufficient to give a reasonably good field topology. At the ends of the wires, there is a four-fold enhancement of the sextupole. Its fringes and those of the dipole extend out about $5 \mathrm{~mm}$ 

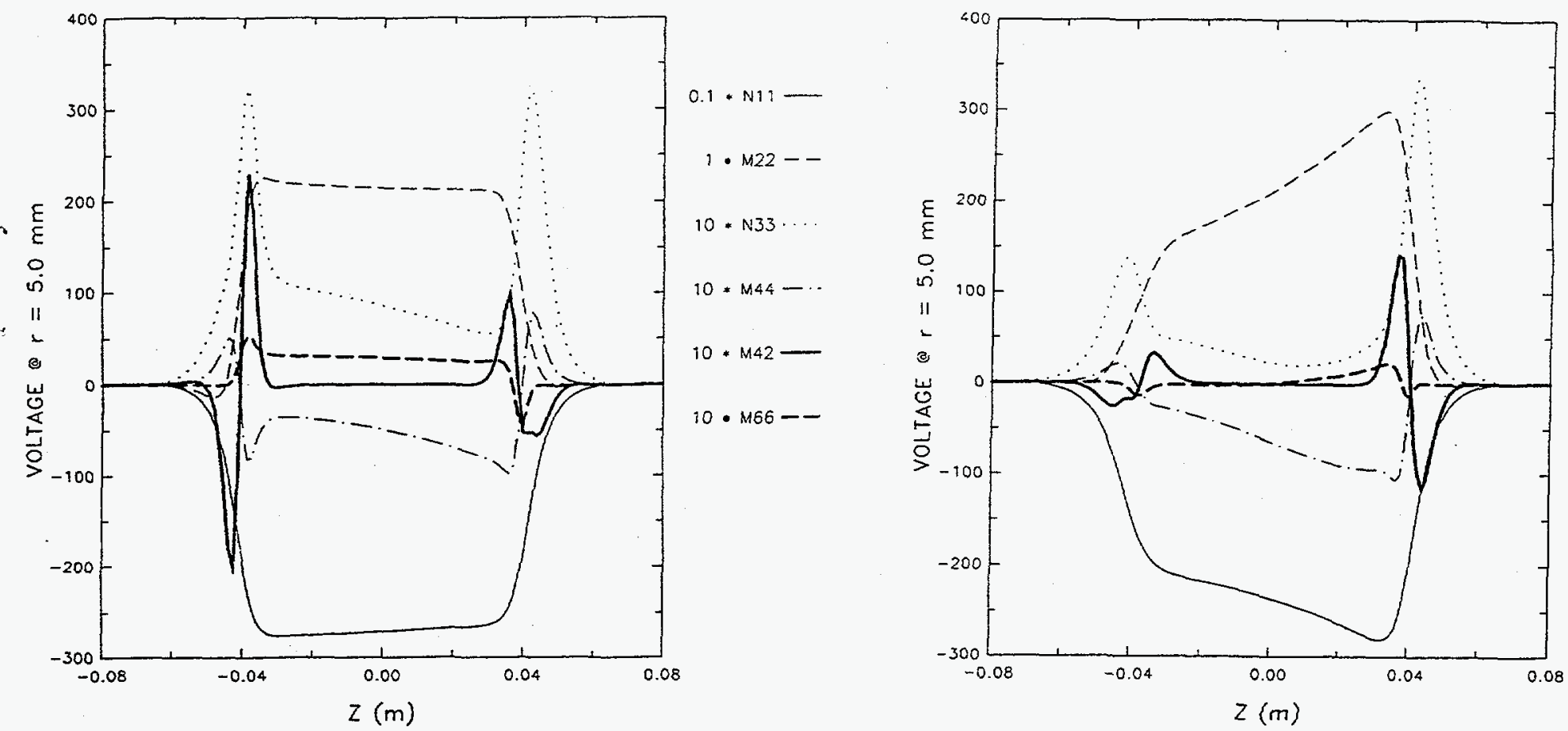

Figure 3: Multipole strength evaluated at $r=5 \mathrm{~mm}$ plotted versus $z$ for the potential distribution in the top cage in an untapered geometry. Note the the dipole has been reduced by a factor of 10 while the sextupole (N33), octupole (M44), and pseudo-octupole (M42) has been multiplied by 10.

further in $z$ than do those of the quadrupole or higher order multipoles. There is also a rather strong pseudo-octupole component (M42) at the upstream entrance to the cage when compared with that predicted (see below) for the tapered geometry.

In Fig. 4 we plot the top cage multipole strength versus $z$ for a tapered geometry with the decomposition being done along a parabolic orbit line passing through the $(x, y, z)$ points $(0.0,16.6,-39 . \dot{3})$ and $(0.0,12.5,39.3) \mathrm{mm}$. With the exception of the sextupole, the $z$-dependent $n$-pole strengths at $r=5 \mathrm{~mm}$ appear to scale as the inverse $n$-th power of the local cage radius; i.e. the higher order multipoles are suppressed at the upstream end of the cage relative to their values at the downstream end. It is interesting (and not understood) that the dodecapole (M66) has odd symmetry with respect to the cage center at $z=0$ whereas in the untapered case (Fig. 3) it has a nearly constant, positive value. The odd symmetry is not repeated in the right cage multipole decomposition (see Fig. 5) where M66 is generally positive and has a larger amplitude than in the untapered case (not shown). Moreover, the sextupole component of the right cage is much stronger than that of the top. This may be because the right cage has fewer electrodes at large $x$ than does the top cage at large $y$ or because of some other (but non-obvious) difference in the electrode topography. It does not appear

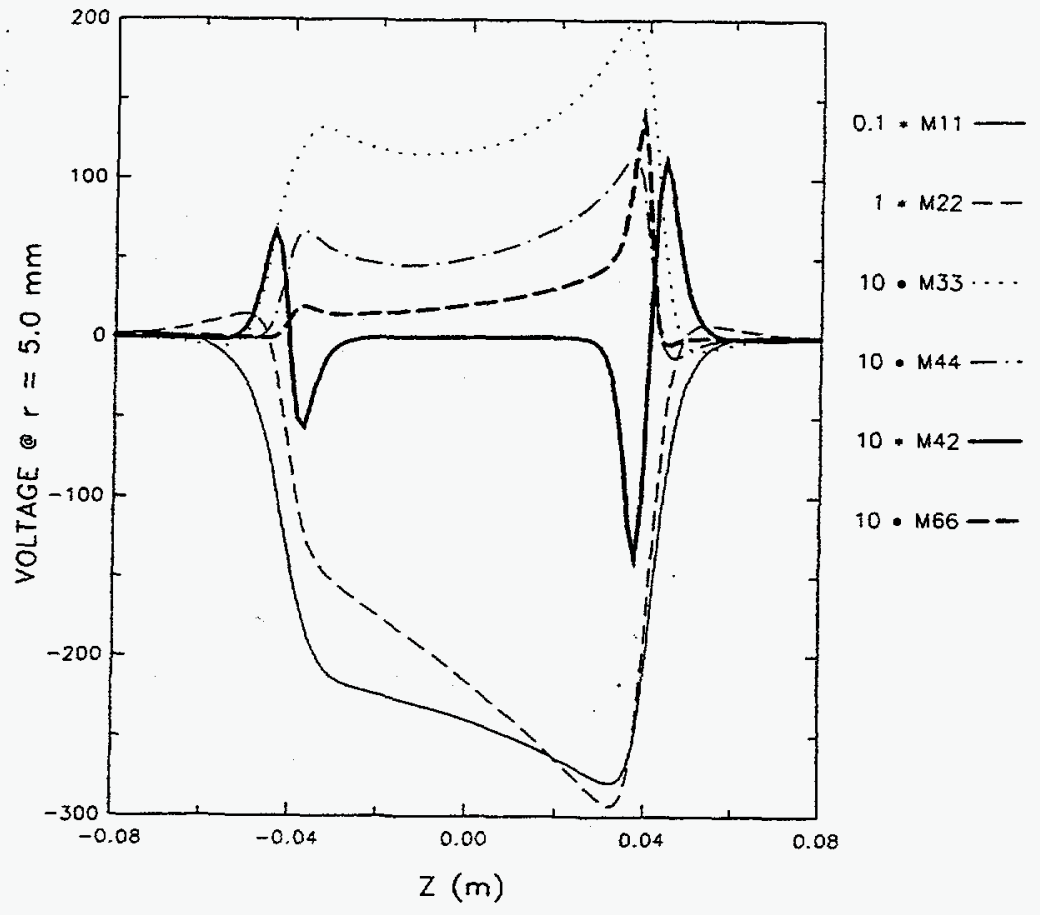

Figure 5: Multipole strength evaluated at $r=5 \mathrm{~mm}$ plotted versus $z$ for the potential distribution in the right cage for a tapered geometry. As in Fig. 3, the sextupole and other anharmonic components have been enhanced relative to the quadrupole and dipole. 


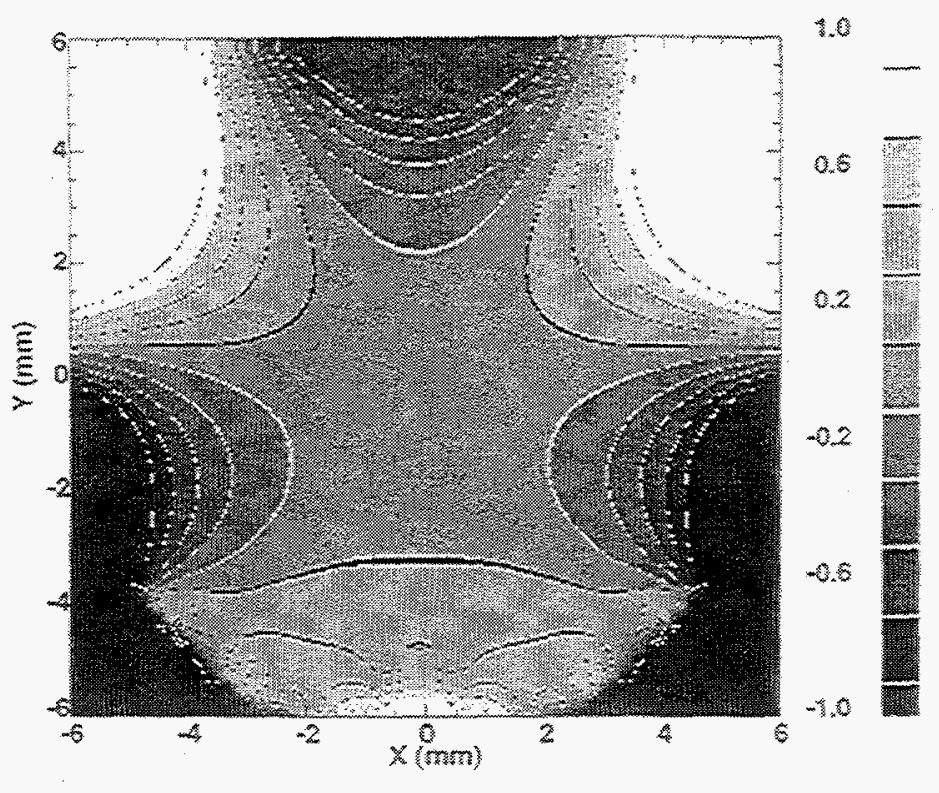

Figure 6: $z$-integrated nonlinear potential (see Eq. 18) in $\mathrm{V}-\mathrm{m}$ for the top cage as a function of the transverse offset from a parabolic path through the top cage. The contour spacing is $0.2 \mathrm{~V}$-m. Values exceeding $\pm 1 \mathrm{~V}$-m have been clipped.

to be due to the taper itself as the untapered geometry also produces a large, positive sextupole component in the right cages.

\section{Predicted Emittance Growth due to Anharmonic Field Com- ponents}

Once one has the harmonic portion of the electrostatic potential (see Eq. 17) from the multipole decomposition, one can numerically determine the integral $\int \Phi_{N L} d z$ along a given path of the beam centroid. If one presumes that the offsets $\left(\Delta x_{i}, \Delta y_{i}\right.$ ) of individual particles from the beam centroid remain constant during transport through the cage (i.e.impulse approximation) and also neglects any "energy effect" with the squirrel-cage due to the transverse-position dependence of an ion's energy when it enters the cage $(\approx 1 \%$ effect), one may use this integral to estimate the associated emittance growth.

In Figs. 6 and 7 we portray this integral as a function of transverse offset from the (presumed parabolic) centroid path for the top and right cages respectively. The plotted values are clipped at \pm 1 volt-m in order to emphasize the topography of $\Phi_{N L}$ in the central region $r \leq 4 \mathrm{~mm}$. An obvious sextupole dominates the core regions of both figures but, to keep things in perspective, its absolute magnitude is small compared to a typical dipole field of $120 \mathrm{~V}$-m (i.e. $40 \mathrm{kV} \times 3 \mathrm{~mm}$ ).

To estimate the RMS emittance growth $\Delta \varepsilon^{2}$, we use the net anharmonic impulse given a particle at offset

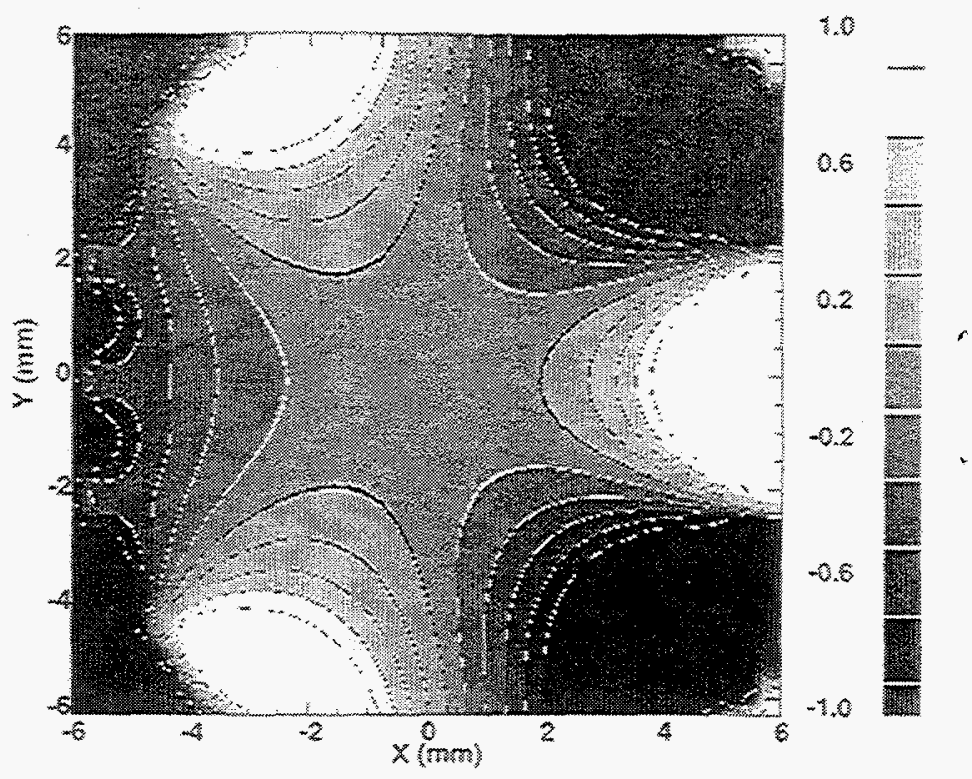

Figure 7: Same as Fig. 6 but for the right cage.

$(\Delta x, \Delta y)$ from the beam centroid:

$$
\delta \mathbf{x}_{i}^{\prime} \approx \frac{-e}{2 V_{B}} \vec{\nabla} \int d z^{\prime} \Phi_{N L}\left(x_{c}+\Delta x_{i}, y_{c}+\Delta y_{i}, z^{\prime}\right)
$$

If we presume that each individual beam has a transverse density profile corresponding to a uniformly filled, upright ellipse with major axis $a$ and minor axis $b$,

$$
\begin{array}{r}
\Delta \varepsilon_{x x^{\prime}}^{2}=\left(<x^{2}>-<x>^{2}\right) \times\left(<x^{\prime 2}>-<x^{\prime}>^{2}\right)- \\
\left(<x x^{\prime}>-<x><x^{\prime}>\right)^{2}
\end{array}
$$

where the average over configuration space is

$$
<f>\equiv \frac{1}{\pi a b} \int_{-a}^{+a} d x \int_{-y_{m}(x)}^{y_{m}(x)} d y f(x, y)
$$

and $y_{m}(x) \equiv(b / a) \times\left(a^{2}-x^{2}\right)^{1 / 2}$.

In order to get a feeling for the usable aperture of the cage, we computed $\Delta \varepsilon^{2}$ as a function of a keeping the ellipticity ratio fixed at a constant value of 1.75 corresponding to the predicted, downstream matched beam size of 6.5 versus $3.7 \mathrm{~mm}$. Figure 8 plots the resultant edge emittance growth where $\varepsilon_{\text {edge }} \equiv 4\left(\Delta \varepsilon^{2}\right)^{1 / 2}$ which should be compared with the initial value of $11.2 \mathrm{~mm}-\operatorname{mrad}$ (equivalent to a normalized edge emittance of $2 \times 10^{-8} \mathrm{~m}-\mathrm{rad}$ ). One sees that for major axis values below about $5 \mathrm{~mm}$, the predicted emittance increase is smaller than the initial emittance while for values exceeding $\approx 6 \mathrm{~mm}$, the increase dominates the initial emittance. Not surprisingly, the emittance degradation is worse in the plane corresponding to the major axis elongation in the top and right cages. Although the corresponding thermal angles remain small (with typical values of $3 \mathrm{mrad}$ or less) when compared to the overall dipole deflection of approximately $100 \mathrm{mrad}$, they are not negligibly small when compared with the initial thermal angle of about 2 mrad. 


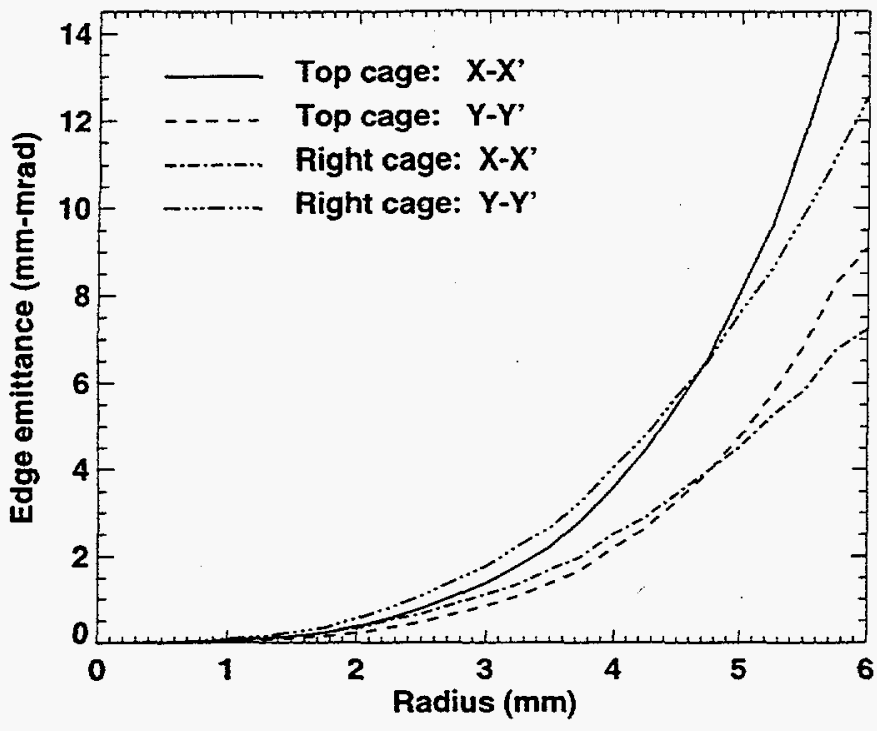

Figure 8: Change in edge emittance in the two transverse planes for an initially cold beam versus beam size for the tapered top and right cages. The beam is presumed to be a uniformly filled, upright ellipse with a major-to-minor axis ratio of 1.75 , with a horizontal(vertical) orientation in the top(right) cage respectively.

\section{Comparison with Earlier Studies and Conclusions}

D. Judd[4] has written an interesting note estimating the fringe fields of the squirrel-cage by considering the impulse force due to a single wire at a constant voltage. For voltages typical of the squirrel-cage, he estimated that the resultant angular deflection due to fringe fields at the wire ends would be or order $1 \mathrm{mrad}$ or less. He predicted a similar value in the neighborhood of two adjacent rods of differing voltages. Since this angle is quite small compared to the $100 \mathrm{mrad}$ dipole deflection angle, he concluded that the fringe fields "may be regarded as negligible".

Three comments are in order here. First, it is comforting that both Judd and myself find that the anharmonic fringe fields contribute deflection angles of the order of $1 \mathrm{mrad}$ and there are not factors of 10 differences. Second, the interested, alert, and savvy reader should note that Judd's calculation is especially relevant quite close to the individual wires, a region where the my results lose validity because of the discretization of the line charge (see \$2). Conversely, my calculation includes the global ensemble of wires and thus picks up components such as the sextupole which cannot appear in Judd's highly localized solution. Third, as pointed out before, while a $1 \mathrm{mrad}$ angle is negligible compared to the bulk centroid deflection angle, it is not small compared to the $\sim 2$-mrad initial thermal angle.

K. Hahn[5] also wrote a note presenting results of a PIC simulation of beam dynamics in the MBE- 4 beam combiner and merging section. Although there is a statement on p. 6 indicating that the normalized emittance increases nearly ten-fold to $\sim 10^{-7} \mathrm{~m}$-rad due to field aber- rations in the squirrel-cage, it appears that this corresponds to RMS value of the entire four-beam configuration which Hahn correctly points out is rather artificial until the beams actually merge beyond the squirrel-cage. More recent work by Hahn (currently in preparation) suggests that there may be a factor of two increase in the individual beam's emittance due to aberrations during transport in the squirrel-cage. These simulation results have a grid resolution of $\approx 0.2 \mathrm{~mm}$ and include both the highly localized $1 / r$ fields near the individual wires and the "global" (i.e. sextupole-including) fields but not the fringe fields at the cage ends. Hence, it is likely that the true emittance growth in the squirrel-cage will be somewhat greater than found in either Hahn's PIC results or those of the present study.

In summary, we find that the the 3-D anharmonic fields of the squirrel-cage contribute $\sim 10 \mathrm{~mm}$-mrad of unnormalized, edge emittance to an MBE- 4 beamlet with a major axis of $5 \mathrm{~mm}$. The dominant component is the sextupole term although the octupole is also important. While this emittance increase is small compared to the final predicted emittance of the merged beams (see [5]), it is comparable to the initial emittance of the uncombined beamlets. Beam particles that come extremely close $(\leq 1 \mathrm{~mm}$ ) to the individual wires will probably pick up additional deflection which is not resolved in this study (but should be in Hahn's[5] work). If it is deemed important to reduce the emittance growth caused by the squirrel-cage, it may be possible to optimize further the individual wire voltage values in order to reduce the unwanted sextupole and octupole terms.

\section{References}

[1] A. Faltens, "The Combiner Final Half-Quad - Preliminary", HIFAR-407, March, 1994.

[2] K. Hahn, Nucl. Inst. and Meth., A 338, 161 (1993).

[3] M. Berz, W. Fawley, and K. Hahn, Nucl. Inst. and Meth., A 307, 1 (1991).

[4] D. L. Judd, "Some Rough Estimates of Combiner 'Squirrel-Cage' Fringe Field Effects", HIFAR-422, October, 1994.

[5] K. Hahn, "2-Dimensional Simulation Study of MBE-4 Combiner", HIFAR-420, September, 1994. 

LAWRENCE BERKELEY NATIONAL LABORATORY UNIVERSITY OF CALIFORNIA

TECHNICAL \& ELECTRONIC INFORMATION DEPARTMENT

BERKELEY, CALIFORNIA 94720 